\title{
EDITORIAL
}

\section{Are mild/moderate acquired idiopathic aplastic anaemia and low-risk myelodysplastic syndrome one or two diseases or both and how should it/they be treated?}

Leukemia (2016) 30, 2127-2130; doi:10.1038/leu.2016.206; published online 2 September 2016

To answer questions in the title, we need to consider four issues: (1) is it possible to accurately diagnose mild/moderate acquired idiopathic aplastic anaemia versus low-risk myelodysplastic syndromes (MDS). Namely, can these diagnoses be definitively established based on clinical, laboratory and histological features; (2) are these diagnoses mutually-exclusive synchronously or metachronously; (3) what is the best way to make a probabilistic diagnosis of one or the other (or both) disorders; and (4) is it necessary to distinguish between these diagnoses or can we develop a therapy strategy based on pathophysiology rather than on diagnosis and which incorporates uncertainty. We address these issues below.

\section{ARE MILD/MODERATE ACQUIRED IDIOPATHIC APLASTIC ANAEMIA AND/OR LOW-RISK MDS DISTINCT, MUTUALLY- EXCLUSIVE DISORDERS?}

Bone marrow failure, defined as persistently low levels of red blood cells, granulocytes and platelets resulting from decreased haematopoietic stem/progenitor cells (HSPCs), is a common haematologic disorder that can be inherited or acquired (We use the term acquired operationally implying the disease is not congenital [present at birth] without implying aetiology. Whether acquired disorders are inherited or not cannot be known with certainty in many instances. However, with rare exception there is discordance for idiopathic aplastic anaemia in geneticallyidentical twins. Nor is there in increased risk in relatives of affected persons). ${ }^{1}$ Acquired bone marrow failure is termed idiopathic when there is no identified exposure capable of damaging the bone marrow and when there is no relevant nutritional deficiency such as vitamin $B_{12}$ or folate (Identification differs from causality. Not all exposures may be known to the subject and/or the haematologist). Potential aetologies of bone marrow failure are presented in Figure 1.

There are several acquired bone marrow failure syndromes including aplastic anaemia, myelodysplastic syndromes (MDS), paroxysmal nocturnal haemoglobinuria ( $\mathrm{PNH})$, idiopathic cytopaenia of undetermined significance ${ }^{2}$ and acquired amegakarytocytic thrombocytopaenia. ${ }^{3}$ Each disease is diagnosed based on the widely-accepted criteria. However, for the two most common, $\mathrm{mild} /$ moderate acquired idiopathic aplastic anaemia and low-risk MDS, there is substantial overlap in diagnostic criteria such that some persons meet criteria for both. Overlap arises because diagnostic criteria of these diseases were separately defined, sometimes without considering potential shared features including epidemiology, histology, cytogenetics, mutational landscape and other variables.

Diagnostic criteria for acquired idiopathic aplastic anaemia include decreased levels of blood cells (typically of all lineages) and decreased bone marrow cellularity with no dysplastic immature or mature myeloid cells or megakaryocytes. However, erythroid dysplasia is often seen in mild/moderate acquired idiopathic aplastic anaemia. ${ }^{4}$ Diagnostic criteria of low-risk MDS also include decreased levels of blood cells (some or all lineages) and dysplastic immature and mature blood cells without a substantial decrease in bone marrow cellularity, although low bone marrow cellularity can be seen on 15-20\% of persons thought to have MDS. ${ }^{5,6}$ Some persons with MDS have dysplasia in only erythroid cells similar to some persons with acquired idiopathic aplastic anaemia and are termed refractory cytopaenia with uni-lineage dysplasia with or without ring sideroblasts.

Because of this overlap in diagnostic criteria it is not surprising some persons diagnosed as having mild/moderate acquired idiopathic aplastic anaemia by one haematologist are diagnosed as having low-risk MDS by another or even by the same haematologist reviewing the same data on a different occasion (intra- and inter-observer variability or discordance). This is because supposed distinguishing features such as bone marrow cellularity and bone marrow and blood cell dysplasia, especially dysplasia of erythroid cells, are to some extent subjective, confounded and have low intra- and inter-observer concordance. ${ }^{7}$ For example, mild/moderate myelodysplasia most often occurs in older persons where decreased bone marrow cellularity is common and who have no haematological disorder. ${ }^{8}$ Also, mutations in some genes associated with acute myeloid leukaemia (AML) and MDS are found in a substantial proportion of older persons with normal or even clonal haematopoiesis but no haematological abnormality within their remaining lifetime. ${ }^{9-11}$ Confusingly, bone marrow cellularity of some persons with acquired idiopathic aplastic anaemia can appear normal or even increased if a biopsy is done at a site where residual haematopoiesis is increased to compensate for the overall decrease at the other bone marrow sites. ${ }^{12}$

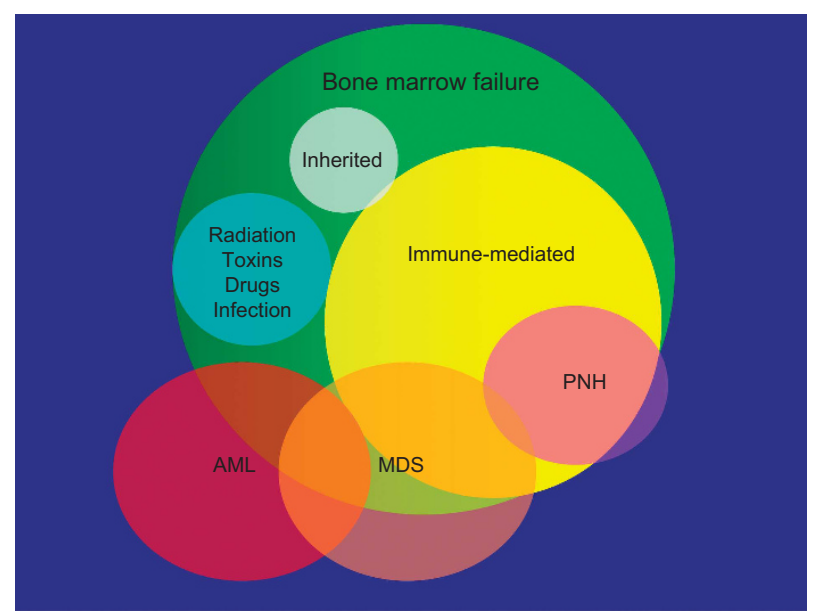

Figure 1. Interrelationship of variables associated with an immune aetiology of bone marrow failure syndrome in persons with normal cytogenetics, no increase in blood or bone marrow blasts and no ring sideroblasts. The precise role of these variables in disease management requires further study. 
Interpretation of cytogenetic abnormalities is also complex and cannot be definitively used to distinguish these disorders. Some persons with monosomy $7(-7)$ or $\operatorname{del}(7 q)$, mild/moderate bone marrow failure and no dysplasia and who likely have aplastic anaemia are diagnosed as having MDS-U according to WHO (World Health Organization) criteria for MDS. ${ }^{13}$ The same confusion exists for persons with del(13q) who are also likely incorrectly diagnosed as having MDS-U although these persons rarely develop $A M L$ and frequently responding to immune suppression. ${ }^{14,15}$

Nevertheless, diagnostic overlap mild/moderate acquired idiopathic aplastic anaemia and low-risk MDS are conceptually very different diseases. Acquired idiopathic aplastic anaemia is usually, but not always, a non-clonal disorder despite a predisposition to neoplastic transformation (typically $\mathrm{MDS}$ and $\mathrm{AML}$ ) whereas MDS is a myeloid neoplasm with a substantial risk of transformation to AML.

\section{WHAT IS THE BEST WAY TO MAKE A PROBABILISTIC DIAGNOSIS OF ONE OR THE OTHER DISORDER?}

Accurately distinguishing mild/moderate acquired idiopathic aplastic anaemia from low-risk MDS without increased blasts or ring sideroblasts is important but at times difficult or impossible. ${ }^{16}$ However, this distinction can be critical because of therapy implications. For example, persons diagnosed as having mild/ moderate acquired idiopathic aplastic anaemia often receive immune suppression whereas persons diagnosed as having low-risk with MDS often receive hypo-methylating drugs. The latter suppress bone marrow function ideally avoided in persons with a non-neoplastic bone marrow disorder. There are, of course, exceptions such as treating young persons with MDS with immune suppression; ${ }^{15-17}$ responses are sometimes observed. However, most haematologists hesitate using anti-thymocyte globulin (ATG) in persons thought to have MDS because of the possibility of worsening bone marrow function and because of the absence of definitive evidence of improved survival. ${ }^{17}$

Can mutation analyses help resolve this diagnostic dilemma? Unfortunately no. Recent studies show overlap in the pattern of mutations in persons with mild/moderate acquired idiopathic aplastic anaemia and low-risk MDS. ${ }^{18,19}$ Whole-exome sequencing of large number of persons with aplastic anaemia identified diverse somatic mutations including DNMT3A and ASXL1 in about $40 \%$. Although similar mutations are detected in persons with MDS and in normal older persons with no haematological disorder or even clonal haematopoiesis, there are some differences in the mutational landscape of persons assumed to have acquired idiopathic aplastic anaemia versus those assumed to have MDS. ${ }^{20}$ Mutations in BCOR/BCORL1 and PIGA are common and associated with a favourable prognosis in persons presumed to have idiopathic aplastic anaemia. ${ }^{18}$ In contrast, mutations in spliceosome genes are common in persons presumed to have MDS but rare in those presumed to have acquired idiopathic aplastic anaemia. ${ }^{18}$ However, no specific mutation is unique to either disease. Consequently, data from genetic analyses may favour one or other diagnosis but are not definitive at the subject-level.

Given these considerations, it is unlikely or impossible a haematologist can precisely determine whether someone with mild/moderate bone marrow failure has acquired idiopathic aplastic anaemia or low-risk MDS. How important is precise estimation? Probably not very, but it is important to have a qualitative estimate, a process that can be considered analogous to an expression of uncertainty such as confidence limit or credibility interval used by frequentist and Bayesian statisticians, respectively. The problem is although statisticians are comfortable with the concept of uncertainty, patients and physicians are not. Both want to know what disease someone has, not what disease they probably have. People told they probably have a disease often find another physician who knows what disease they have. This is understandably satisfying to both parties. But is it true?

\section{CAN WE DEVELOP A THERAPY STRATEGY BASED ON PATHOPHYSIOLOGY RATHER THAN ON DIAGNOSIS AND WHICH INCORPORATES UNCERTAINTY?}

Is there a different way out of this dilemma? We think so. Our approach relies on considering the pathophysiology of the bone marrow failure rather than focusing on a histological diagnosis. Namely, is bone marrow failure from immunemediated destruction of HSPCs or does malfunction of HSPCs result from acquired genetic abnormalities (mutations) which we term pre-leukaemia. Table 1 shows laboratory variables correlated with response to immune interventions such as ATG and/or cyclosporine. Although one could argue a response to ATG is not specific for an immune aetiology of bone marrow failure, response to cyclosporine can be taken to indicate an underlying immune aetiology because its mechanism of action is thought to be restricted to T-cells. ${ }^{21}$ Several other variables such as PNH-type cells are also associated with response to ATG and/or cyclosporine. $^{22-24}$ Another potential marker of an immunemediated aetiology for bone marrow failure is the presence of leukocytes lacking HLA-A alleles (HLA-LLs). ${ }^{25,26}$ These cells develop from HSPCs with copy number-neutral loss of heterozygosity of the HLA-class I allele(s) owing to 6pUPD (uni-parental disomy of the short arm of chromosome 6). ${ }^{25}$ It is important to consider these clones are present in normal bone marrow before immunemediated bone marrow failure develops and are selected because of the failure of non-mutated HSPCs. ${ }^{27}$ Another potentially useful correlate of response to immune suppression is high plasma levels of thrombopoietin often associated with increased numbers of PNH-type cells. ${ }^{28}$

Table 1. Variables potentially useful in estimating aetiology of bone marrow failure

\begin{tabular}{|c|c|c|c|}
\hline & Immune-mediated & Equivocal & Pre-leukemia \\
\hline Increased percentage of PNH-type cells & $(+)$ & $(-)$ & $(-)$ \\
\hline HLA-class I allele-lacking leukocytes & $(+)$ & $(-)$ & $(-)$ \\
\hline $\begin{array}{l}\text { Increased percentages of bone marrow } \\
\text { CD } 34^{+} \text {or } \mathrm{CD} 42 \mathrm{~b}^{+} \text {cells }\end{array}$ & $(-)$ & $(-)$ & $(+)$ \\
\hline HLA-DR15 (ref. 29) & $(+)$ & $(+)$ or $(-)$ & $(+)$ or $(-)$ \\
\hline
\end{tabular}




\section{CONCLUSION}

Where does this leave us? As we discuss, it is impossible to definitively distinguish these conditions and the best approach is probabilistic. Moreover, there is no reason to think a person with $\mathrm{mild} /$ moderate aplastic anaemia might not progress to low-risk MDS. Some aetiological agents such as ionizing radiations, benzene and chloramphenicol cause both disorders. Consequently, there is no scientific basis to exclude the possibility of a person evolving from one to the second disorder or developing both synchronously or metachronously.

We suggest that haematologists be cautious declaring someone with mild/moderate bone marrow failure has low-risk MDS rather than acquired idiopathic aplastic anaemia and the contrary. Rather they should focus on the weight of evidence favouring an immune-mediated versus genetic abnormality. Review of bone marrow samples by additional expert haematopathologists is unlikely to resolve this issue (Often there is discordance, even among expert haematopathologists in the borderline cases. More importantly, lack certainty of which diagnosis is correct, consensus does not guarantee accuracy.). ${ }^{25} \mathrm{~A}$ reasonable strategy in persons with variables favouring an immune aetiology and/or response to immune suppression (PNH-type cells, HLA-LLs, high thrombopoietin levels) could receive immune suppression first regardless of which diagnosis one thinks is most likely correct. They may have either, both or neither, namely, something else. Other interventions such as hypo-methylating drugs, transplants or investigational interventions could be used in persons failing to respond to immune suppression, responding but then relapsing or when their disease evolves to more closely resemble MDS.

Finally, haematologists need to become more comfortable with the notion of diagnostic certainty, or more accurately, uncertainty. Perhaps new data will rescue us from this conundrum. So, to return to the question we began with: are mild/moderate acquired idiopathic aplastic anaemia and low-risk MDS one or two disease or both? Both we think, at least sometimes and in some people. Francis Bacon said: Without certainty, science is nothing more than seemingly sophisticated guesswork. True. But we are practicing medicine, not science. Get over it.

\section{CONFLICT OF INTEREST}

RPG is a part-time employee of Celgene Corp.

\section{ACKNOWLEDGEMENTS}

Profs John Bennett (Univ. Rochester), Peter Greenberg (Stanford Univ.), Mario Cazzola (Univ. Pavia), Inderjeet Dokal (Queen Mary Univ. of London), Masao Tomonaga (Nagasaki Global Citizens Assembly for Elimination of Nuclear Weapons) and Yasushi Miyazaki (Nagasaki Univ.) kindly reviewed the typescript. SN acknowledges support from JSPS Grants-in-Aid for Scientific Research (KAKENHI). RPG acknowledges support from the National Institute of Health Research (NIHR) Biomedical Research Centre funding scheme.

S Nakao ${ }^{1}$ and RP Gale ${ }^{2}$

${ }^{1}$ Department of Haematology, Faculty of Medicine, Kanazawa University Institutes of Medical, Pharmaceutical, and Health Sciences, Kanazawa, Japan and

${ }^{2}$ Division of Experimental Medicine, Department of Medicine, Haematology Research Centre, Imperial College London, London, UK E-mail: robertpetergale@alumni.ucla.edu

\section{REFERENCES}

1 Young NS, Calado RT, Scheinberg P. Current concepts in the pathophysiology and treatment of aplastic anemia. Blood 2006; 108: 2509-2519.

2 Wimazal F, Fonatsch C, Thalhammer R, Schwarzinger I, Mullauer L, Sperr WR et al. Idiopathic cytopenia of undetermined significance (ICUS) versus low risk MDS: the diagnostic interface. Leuk Res 2007; 31: 1461-1468.
3 Hoffman R, Bruno E, Elwell J, Mazur E, Gewirtz AM, Dekker P et al. Acquired amegakaryocytic thrombocytopenic purpura: a syndrome of diverse etiologies. Blood 1982; 60: 1173-1178.

4 Killick SB, Bown N, Cavenagh J, Dokal I, Foukaneli T, Hill A et al. Guidelines for the diagnosis and management of adult aplastic anaemia. Br J Haematol 2016; 172: 187-207.

5 Tuzuner N, Cox C, Rowe JM, Watrous D, Bennett JM. Hypocellular myelodysplastic syndromes (MDS): new proposals. Br J Haematol 1995; 91: 612-617.

6 Bennett JM, Orazi A. Diagnostic criteria to distinguish hypocellular acute myeloid leukemia from hypocellular myelodysplastic syndromes and aplastic anemia: recommendations for a standardized approach. Haematologica 2009; 94: 264-268.

7 Della Porta MG, Travaglino E, Boveri E, Ponzoni M, Malcovati L, Papaemmanuil E et al. Minimal morphological criteria for defining bone marrow dysplasia: a basis for clinical implementation of WHO classification of myelodysplastic syndromes. Leukemia 2015; 29: 66-75.

8 Parmentier S, Schetelig J, Lorenz K, Kramer M, Ireland R, Schuler U et al. Assessment of dysplastic hematopoiesis: lessons from healthy bone marrow donors. Haematologica 2012; 97: 723-730.

9 Genovese G, Kahler AK, Handsaker RE, Lindberg J, Rose SA, Bakhoum SF et al. Clonal hematopoiesis and blood-cancer risk inferred from blood DNA sequence. N Engl J Med 2014; 371: 2477-2487.

10 Jaiswal S, Fontanillas P, Flannick J, Manning A, Grauman PV, Mar BG et al. Age-related clonal hematopoiesis associated with adverse outcomes. N Engl J Med 2014; 371: 2488-2498.

11 Xie M, Lu C, Wang J, McLellan MD, Johnson KJ, Wendl MC et al. Age-related mutations associated with clonal hematopoietic expansion and malignancies. Nat Med 2014; 20: 1472-1478.

12 Nishimura R, Mase S, Araki R, Fujiki T, Kuroda R, Maeba $\mathrm{H}$ et al. Massive hyperreactive hematopoietic nests in bilateral iliac bones in a patient with mild aplastic anemia. Pediatr Blood Cancer 2014; 61: 1903-1904.

13 Vardiman JW, Thiele J, Arber DA, Brunning RD, Borowitz MJ, Porwit A et al. The 2008 revision of the World Health Organization (WHO) classification of myeloid neoplasms and acute leukemia: rationale and important changes. Blood 2009; 114: 937-951.

14 Hosokawa K, Katagiri T, Sugimori N, Ishiyama K, Sasaki Y, Seiki Y et al. Favorable outcome of patients who have $13 q$ deletion: a suggestion for revision of the WHO 'MDS-U' designation. Haematologica 2012; 97: 1845-1849.

15 Holbro A, Jotterand M, Passweg JR, Buser A, Tichelli A, Rovo A. Comment to "Favorable outcome of patients who have $13 q$ deletion: a suggestion for revision of the WHO 'MDS-U' designation" Haematologica. 2012;97(12):1845-9. Haematologica 2013; 98: e46-e47.

16 Yamazaki $\mathrm{H}$, Nakao S. Border between aplastic anemia and myelodysplastic syndrome. Int J Hematol 2013; 97: 558-563.

17 Passweg JR, Giagounidis AA, Simcock M, Aul C, Dobbelstein C, Stadler M et al. Immunosuppressive therapy for patients with myelodysplastic syndrome: a prospective randomized multicenter phase III trial comparing antithymocyte globulin plus cyclosporine with best supportive care--SAKK 33/99. J Clin Oncol 2011; 29: 303-309.

18 Yoshizato T, Dumitriu B, Hosokawa K, Makishima H, Yoshida K, Townsley D et al. Somatic mutations and clonal hematopoiesis in aplastic anemia. $N$ Engl J Med 2015; 373: 35-47.

19 Kulasekararaj AG, Jiang J, Smith AE, Mohamedali AM, Mian S, Gandhi S et al. Somatic mutations identify a subgroup of aplastic anemia patients who progress to myelodysplastic syndrome. Blood 2014; 124: 2698-2704.

20 Ogawa S. Clonal hematopoiesis in acquired aplastic anemia. Blood 2016; 128: 337-347.

21 Nimer SD, Golde DW, Kwan K, Lee K, Clark S, Champlin R. In vitro production of granulocyte-macrophage colony-stimulating factor in aplastic anemia: possible mechanisms of action of antithymocyte globulin. Blood 1991; 78: 163-168.

22 Sugimori C, Chuhjo T, Feng X, Yamazaki H, Takami A, Teramura M et al. Minor population of CD55-CD59- blood cells predicts response to immunosuppressive therapy and prognosis in patients with aplastic anemia. Blood 2006; 107: 1308-1314.

23 Kulagin A, Lisukov I, Ivanova M, Golubovskaya I, Kruchkova I, Bondarenko S et al. Prognostic value of paroxysmal nocturnal haemoglobinuria clone presence in aplastic anaemia patients treated with combined immunosuppression: results of two-centre prospective study. Br J Haematol 2014; 164: 546-554.

24 Tutelman PR, Aubert G, Milner RA, Dalal BI, Schultz KR, Deyell RJ. Paroxysmal nocturnal haemoglobinuria phenotype cells and leucocyte subset telomere length in childhood acquired aplastic anaemia. Br J Haematol 2014; 164: 717-721.

25 Katagiri T, Sato-Otsubo A, Kashiwase K, Morishima S, Sato Y, Mori Y et al. Frequent loss of HLA alleles associated with copy number-neutral $6 \mathrm{pLOH}$ in acquired aplastic anemia. Blood 2011; 118: 6601-6609. 
26 Maruyama H, Katagiri T, Kashiwase K, Shiina T, Sato-Otsubo A, Zaimoku Y et al. Clinical significance and origin of leukocytes that lack HLA-A allele expression in patients with acquired aplastic anemia. Exp Hematol 2016; e-pub ahead of print 29 May 2016; doi: 10.1016/j.exphem.2016.05.013.

27 Mochizuki K, Sugimori C, Qi Z, Lu X, Takami A, Ishiyama K et al. Expansion of donorderived hematopoietic stem cells with PIGA mutation associated with late graft failure after allogeneic stem cell transplantation. Blood 2008; 112: 2160-2162.
28 Seiki Y, Sasaki Y, Hosokawa K, Saito C, Sugimori N, Yamazaki H et al. Increased plasma thrombopoietin levels in patients with myelodysplastic syndrome: a reliable marker for a benign subset of bone marrow failure. Haematologica 2013; 98: 901-907.

29 Sloand EM, Olnes MJ, Shenoy A, Weinstein B, Boss C, Loeliger K et al. Alemtuzumab treatment of intermediate- 1 myelodysplasia patients is associated with sustained improvement in blood counts and cytogenetic remissions. J Clin Oncol 2010; 28: 5166-5173. 